\title{
Repeat Surgery in Chronic Aortic Dissection: A New Technique without Touching the Native Aorta
}

\author{
Gian Luca Martinelli, MD ${ }^{1}$ Attilio Cotroneo, $\mathrm{MD}^{1}$ Valerio Tolva, $\mathrm{MD}^{2}$ Felice Armienti, $\mathrm{MD}^{3}$ \\ Mario Bobbio, MD ${ }^{1}$ Gabriele Musica, MD ${ }^{1}$ Enrico Visetti, MD ${ }^{4}$ Ugo Filippo Tesler, MD ${ }^{1}$ \\ ${ }^{1}$ Department of CardioVascular, Clinica San Gaudenzio-Gruppo \\ Policlinico di Monza, Novara, Italy \\ 2 Department of Vascular Surgery, Policlinico di Monza, Monza, Italy \\ 3 Department of Radiology, Clinica San Gaudenzio-Gruppo Policlinico \\ di Monza, Novara, Italy \\ ${ }^{4}$ Department of Anesthesia, Clinica San Gaudenzio-Gruppo \\ Policlinico di Monza, Novara, Italy \\ Address for correspondence Gian Luca Martinelli, MD, Via Oslavia 50, \\ Rome 00195, Italy (e-mail: martinelligluca@gmail.com). \\ AORTA 2019;7:163-168.
}

\begin{abstract}
Keywords

- aortic dissection

- repeat surgery

- frozen elephant trunk technique

Background Repeat surgery of the chronically dissected aorta following repair of a Type-A acute aortic dissection (AAD) still represents a challenge. The proposed surgical options are as follows: (1) staged procedure with elephant trunk (ET) technique, (2) traditional frozen elephant trunk (FET) intervention, and (3) beating heart cerebral vessel debranching followed by thoracic endovascular aortic repair (TEVAR). However, a marked enlargement of the proximal descending thoracic aorta might make it difficult to perform FET/ET intervention. Furthermore, because in conventional surgery for $A A D$, a prosthetic graft replacement is generally limited to the ascending aorta, and in repeat surgery, this short Dacron graft rarely provides enough room to allow a beating heart cerebral vessel debranching and obtaining a reliable landing zone for the implantation of a firmly anchored stent graft.

Methods We retrospectively reviewed all the five consecutive patients treated in our institution, between 2014 and 2017, for chronic aortic dissection after successful surgical treatment of acute Type-A aortic dissection with graft replacement limited to the ascending aorta. The five patients underwent repair utilizing a modified FET technique with total aortic arch and upper descending aorta exclusion without touching the native dissected aorta.

Results No early- or midterm mortality was observed. Mean time interval between the initial and the reoperative procedure was 26 months (range, 3-80 months). No patient had a minor/major neurologic event. Mean circulatory arrest time was 16 minutes (range, 11-25 minutes). Mean follow-up time was 22 months (range, 9-42 months).

Conclusions We report our initial experience with a modified FET technique realized by anastomosing the stent graft with the previously implanted ascending aortic graft in Hishimaru's zone 0 and by rerouting all cerebral vessels without "touching" the native chronically dissected aorta. A larger number of patients and a longer follow-up will be required to confirm these initial encouraging results.
\end{abstract}

received

April 24, 2018 accepted after revision November 2, 2019
DOI https://doi.org/

10.1055/s-0039-3402071. ISSN 2325-4637.
Copyright @ 2019 by Thieme Medical Publishers, Inc., 333 Seventh Avenue, New York, NY 10001, USA. Tel: +1(212) 760-0888.
License terms

(1) (1) 


\section{Introduction}

During conventional surgery for acute Type-A aortic dissection (AAD), a prosthetic replacement limited to the ascending aorta is generally performed, without cerebral vessel debranching and/or arch replacement. ${ }^{1,2}$ Aneurysmal dilatation of the residual dissected aorta may occur, particularly if the intimal tear involved the aortic arch at the time of the initial operation. ${ }^{3-5}$ The need for reoperation is greater in patients with Marfan's syndrome. ${ }^{6}$ For this reason, some patients may require surgical repair of the chronically dissected aortic arch and the descending thoracic aorta.

Staged procedures for management of extensive disease of the remaining thoracic aorta, including the use of the elephant trunk (ET) technique, have been successfully used to treat the aforementioned conditions. ${ }^{7-10}$. Although these are useful techniques, marked aneurysmal dilatation of the proximal descending thoracic aorta (the usual site for anchoring of the aortic graft) is often present in the setting of chronic aortic dissection (ChAD) marking that dilation may preclude the safe anastomosis of a graft to the aorta in this area. Furthermore, the aggregate mortality and morbidity for the staged procedure may be substantial.

A less invasive approach may be represented by a hybrid approach with cerebral vessel rerouting followed by a TEVAR. But the limited length of the previously implanted ascending aortic graft rarely provides enough room allowing a beatingheart cerebral vessel debranching and the creation of a reliable landing zone for the implantation of a firmly anchored thoracic endovascular stent graft. ${ }^{11-13}$

In the last few years, many publications have advocated the advantage of the FET technique which combines the cutand-sew technique at the level of the distal aortic arch with an endovascular stent-graft implantation in the descending thoracic aorta. ${ }^{14-17}$ As FET has evolved, many variations of this procedure have been described. All these procedures have in common that the stent graft is delivered into the open aorta under circulatory arrest and it is sutured, at least partially, into the dissected aortic arch. This intervention is a major surgical procedure, requiring prolonged time on cardiopulmonary bypass (CPB), hypothermic circulatory arrest, and selective cerebral perfusion. The prolonged spinal cord ischemia, associated with the exclusion of several intercostal arteries, increases the risk of paraplegia. In the Bologna experience, Di Eusanio et $\mathrm{al}^{18}$ described an incidence of $7.4 \%$ permanent neurologic dysfunction and $9.0 \%$ spinal cord injury. Further, the execution of the distal anastomosis in Hishimaru's zone 3 may be impaired by its distal location and by the frequent poor quality of the dissected aortic wall in this area.

We propose a new technique which preserves all the advantages of FET. Anastomosing the stent graft to the previously implanted ascending aortic graft allows complete exclusion of the aortic arch and of the proximal thoracic descending aorta without "touching" the native chronically dissected aorta. Since 2014, we have employed this technique in five patients without observing any early or midterm mortality or any major neurological complications.

\section{Materials and Methods}

We retrospectively analyzed all consecutive patients treated in our institution between 2014 and 2017 for ChAD after the successful surgical treatment of acute Type-A aortic dissection with graft replacement of the ascending aorta.

Patients in whom cerebral vessels rerouting had been performed at the first operation have been excluded from this report. They were treated by traditional thoracic endovascular aneurysm repair (TEVAR) at the repeat operation. Furthermore, when the previous ascending aorta graft implant was longer than $5 \mathrm{~cm}$, cerebral vessel debranching on beating heart was performed to obtain a reliable landing zone for allowing a stable anchored stent graft for TEVAR. Also, this group of patients was excluded from this report.

The institutional review board approved this study. All patients provided written consent.

Five patients underwent surgical correction of the chronic dissection with our modified FET technique with total aortic arch and upper descending aortic exclusion without touching the native aorta. The same surgeon performed all procedures. The indication for surgery was aneurysmal enlargement of a ChAD involving the aortic arch and the descending thoracic aorta. The choice of this technique was related to the presence of a short previous ascending aortic graft $(<5 \mathrm{~cm})$. We used a C-Tag stent graft (C-TAG, WLGore Arizona) of 31 of $34 \mathrm{~mm}$ diameter and $20-\mathrm{cm}$ length in all patients.

Patient characteristics are described in -Table 1. Mean age was $52 \pm 15$ years, and $80 \%$ were men. There were two Marfan's patients. All patients were in NYHA class 1 to 2. Mean delay time between first operation for acute aortic dissection and the second operation was 26 months (range, 3-80 months).

\section{Surgical Technique}

Preoperative computed tomography (CT) of the chest, abdomen, and pelvis is performed to assess surgical strategy and to determine the stent-graft distal landing zone. The selection of the stent graft is based on the diameter of the previously inserted graft in the ascending aorta with an oversizing of 2 to $4 \mathrm{~mm}$. As an example, in a 28 of $30-\mathrm{mm}$ graft in the ascending aorta, we generally insert a $34-\mathrm{mm}$ stent-graft. Such selection proves adequate for stenting the descending aortic aorta.

Table 1 Baseline patient characteristics

\begin{tabular}{|l|l|l|}
\hline Characteristics & $\mathbf{n = 5}$ & $\%$ \\
\hline Age (y) & $52 \pm 15$ & - \\
\hline Male & 4 & 80 \\
\hline $\begin{array}{l}\text { Left ventricular ejection } \\
\text { fraction }>50 \%\end{array}$ & 5 & 100 \\
\hline NYHA class I-II & 5 & 100 \\
\hline Elective surgery & 5 & 100 \\
\hline Previous surgery for AAD & 5 & 100 \\
\hline $\begin{array}{l}\text { Interval between } \\
\text { operations (mo) }\end{array}$ & 26 (range, 3-80) & - \\
\hline
\end{tabular}

Abbreviations: AAD, acute aortic dissection; NYHA, New York Heart Association. 
The first step of the procedure consists of performing an 8mm PTFE carotid-left subclavian artery bypass, through a left supraclavicular incision. Pressures are monitored in both radial arteries and in the left femoral artery. Cerebral oxymetry is assessed by the noninvasive INVOS system (Covidien, Medtronic Inc., Minneapolis, MN). Next, an echo-guided pig-tail catheter is threaded though the left femoral artery in the true lumen of the dissected aorta until its tip reaches the lumen of the previously implanted ascending aortic graft.

Median sternotomy is performed and the incision is extended to the left cervical area for ease of control of the cerebral vessels, particularly of the origin of the left subclavian artery. A trifurcated modified graft with a side-arm perfusion port is selected (Hemashield's 12-8-8 mm) and two of its distal branches are respectively anastomosed to the transected left common carotid and to the innominate artery with the heart beating. ${ }^{18}$ During this time, the cerebral vessels are perfused via the native left subclavian artery previously anastomosed to the left carotid artery (-Fig. 1). With sequential debranching and reperfusion of the three head vessels, as described by Matalanis et al, ${ }^{19}$ complete and rapid cerebral perfusion is ensured.

CPB is then started between the right femoral artery and the right atrium. The ascending aorta is crossclamped and antegrade cardioplegia is infused. At this point, the cerebral vessels are perfused by the $\mathrm{CPB}$ in a retrograde fashion

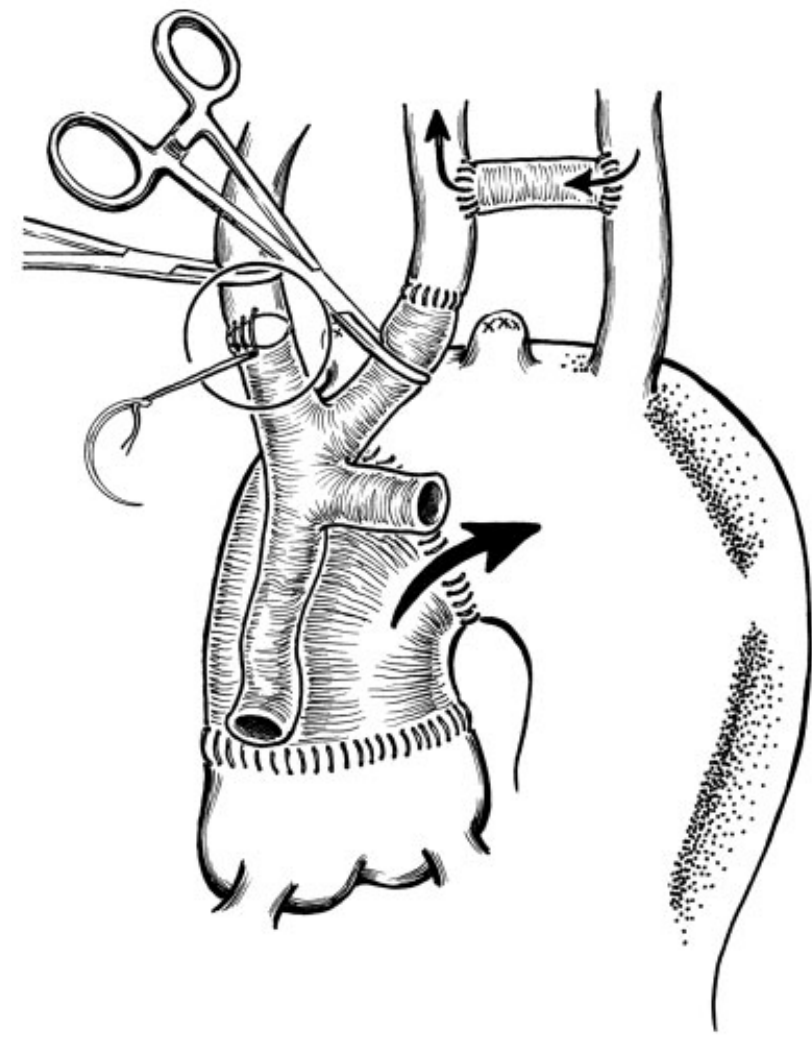

Fig. 1 Carotid-left subclavian artery bypass and innominate and left carotid reimplantation on the trifurcated graft. The arrow indicates the direction of blood flow for cerebral vessels perfusion during all the 4 steps of the operation: anterograde flow, on beating heart, guarantee left subclavian artery and left carotid throw the PTFE carotid-left subclavian artery bypass. through the subclavian artery. The proximal extremity of the trifurcated graft is anastomosed to the graft originally implanted in the ascending aorta (-Fig. 2). When this stage is completed, antegrade perfusion of all cerebral vessels is achieved via the cannulated third-arm perfusion port of the trifurcated graft. The native left-subclavian artery is ligated.

An appropriate stent graft is selected and prepared. The patient is cooled to $28^{\circ} \mathrm{C}$ and CPB is discontinued. Under circulatory arrest, the ascending Dacron graft is opened proximally to the old distal anastomosis. The selected stent graft is prepared. A superstiff wire guide (Lunderquist extra stiff wire guide, Cook Medical LLC, Bloomington, IN) is introduced through the pigtail previously inserted from the left femoral artery and, through this access, the stent graft is deployed in an antegrade fashion under direct vision.

Once the stent graft is deployed, the stent graft is sutured to the original ascending aorta Dacron graft (-Figs. 3-5).

At this point, CPB is restarted. When the rewarming is complete, cardiopulmonary is discontinued.

\section{Results}

There were no intraoperative or in-hospital deaths. - Table 2 describes continuous operative data. The postoperative course was uncomplicated for all patients. All patients were extubated

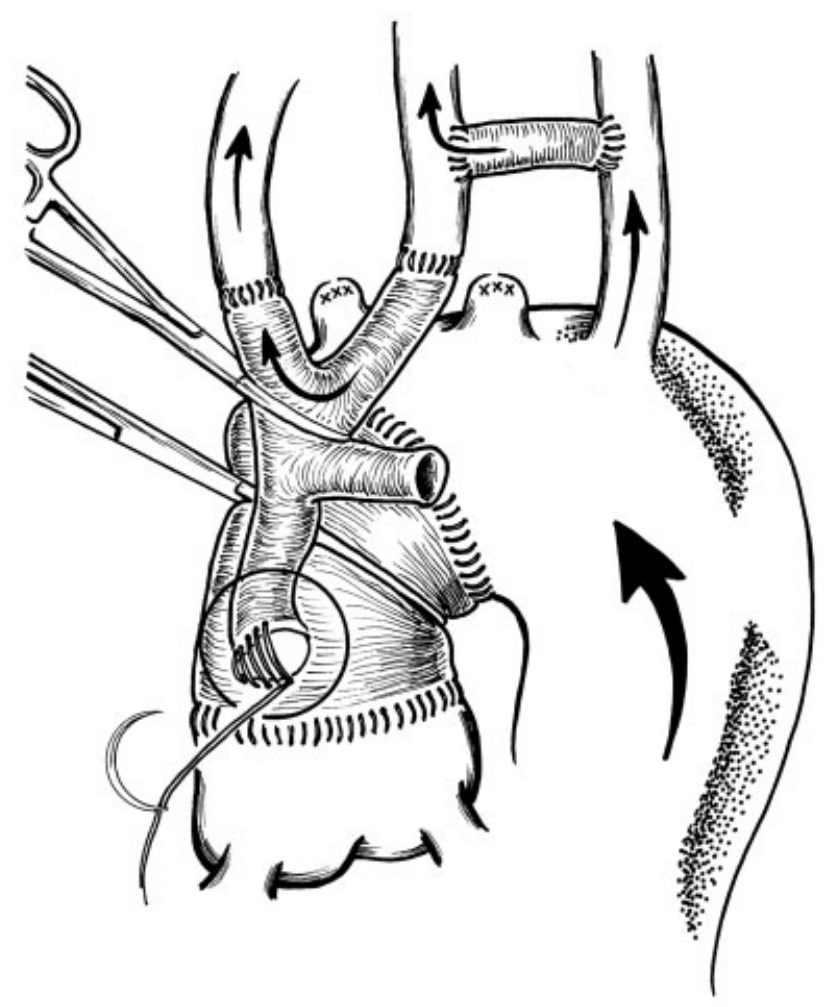

Fig. 2 Trifurcated graft anastomosed proximally on the ascending aorta graft on cardiopulmonary bypass with aorta cross clamped to allow an easy proximal reimplantation close to the previous anastomosis. Retrograde flow from cardiopulmonary bypass throw femoral arterial cannulation. Please note, at this time of the operation, left carotid artery and the innominate artery are perfused throw carotidleft subclavian bypass graft. 


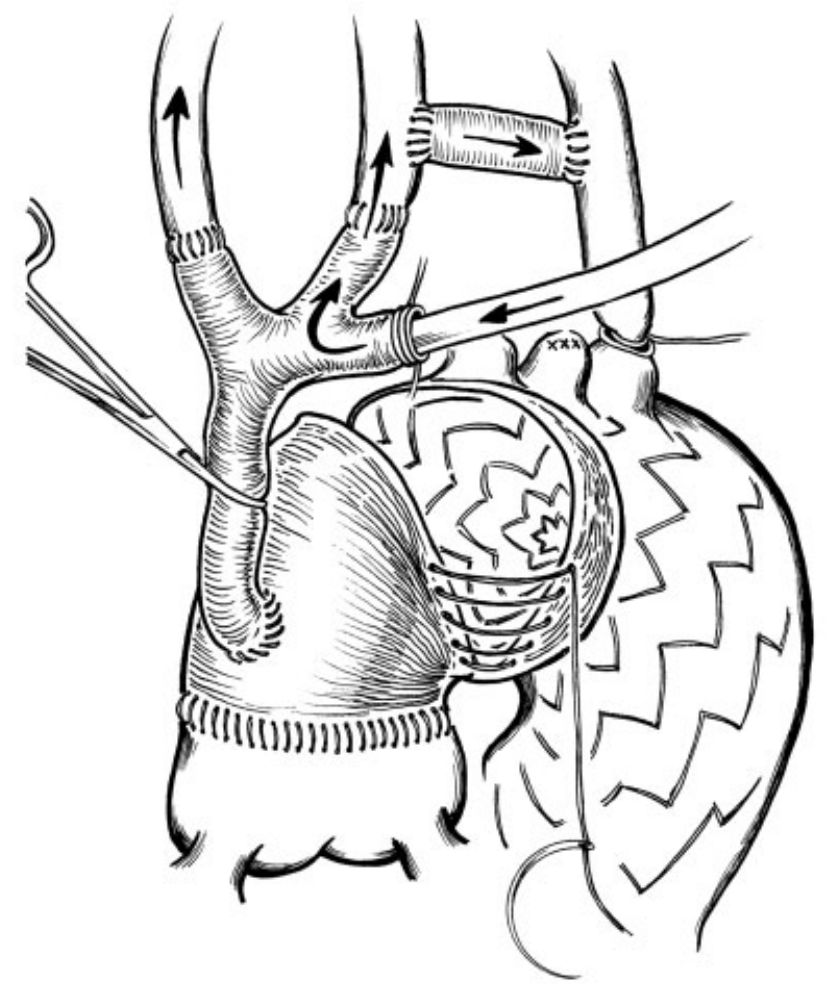

Fig. 3 Stent-graft released under circulatory arrest and fixed to the previously implanted ascending aorta graft. During circulatory arrest time, all three cerebral vessels are continuous perfused by a cannula inserted on the trifurcated modified graft.

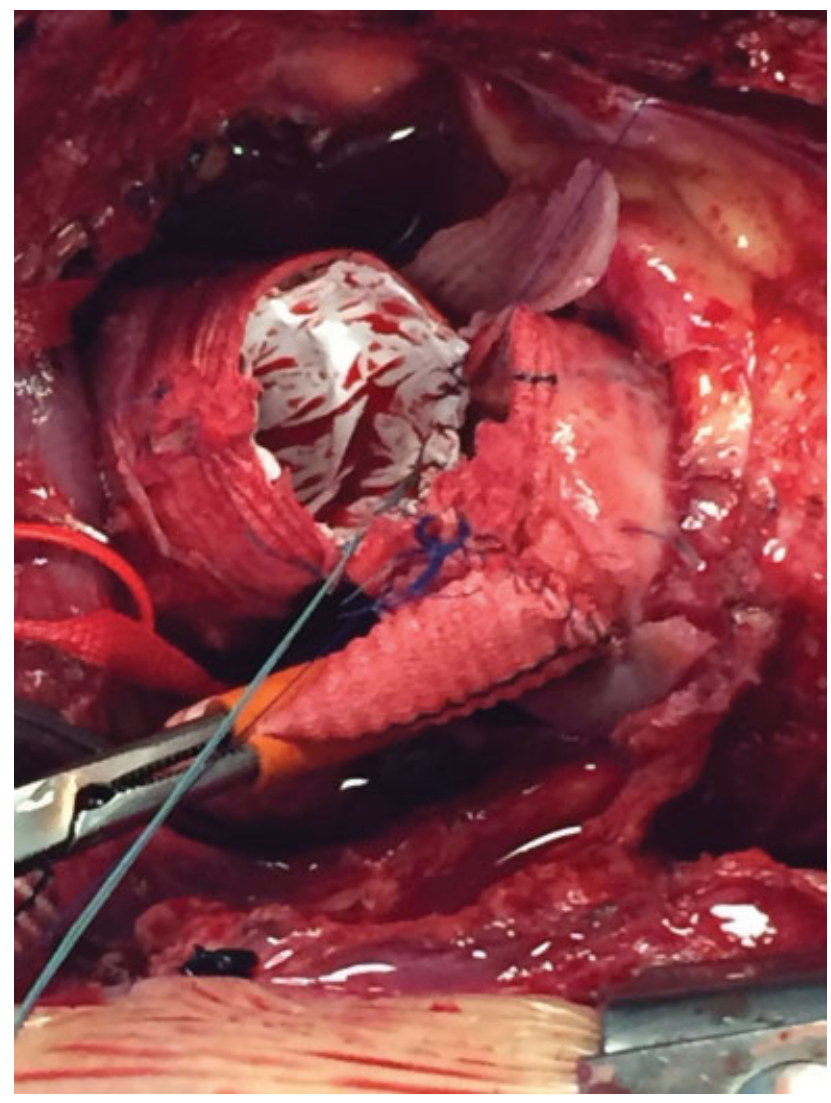

Fig. 4 Intraoperative photograph of stent graft sewing over previous implanted ascending aorta Dacron graft. Reproduced with the permission from photographic archives of Clinica San Gaudenzio, Novara.



Fig. 5 Final result with aortic arch and descending thoracic aorta replacement. At the end of the operation, we remove the clamp on the principal branch of the trifurcated graft and all three cerebral vessels are perfused.

Table 2 Continuous operative data

\begin{tabular}{|l|l|}
\hline Variables (min) & Mean (range) \\
\hline Cardiopulmonary bypass & $141(98-171)$ \\
\hline Cardiac arrest time & $74(54-93)$ \\
\hline Hypothermic circulatory arrest & $16(11-25)$ \\
\hline Selective cerebral perfusion & $31(15-80)$ \\
\hline
\end{tabular}

Note: selective cerebral perfusion was considered the perfusion of the cerebral vessels by the dedicated trifurcated graft; all three cerebral vessels were perfused.

on the first postoperative day and discharged within 15 days. No patient had a minor/major neurologic event.

Follow-up was complete for all patients with a postoperative CT scan (-Fig. 6). Mean follow-up time was 22 months (range, 9-42 months). No mortality was observed. Three patients underwent thoracoabdominal surgery within 3 months from the operation. One patient has a Type-II endoleak due to an incomplete ligation of the subclavian artery that was treated successfully by coil embolization. 


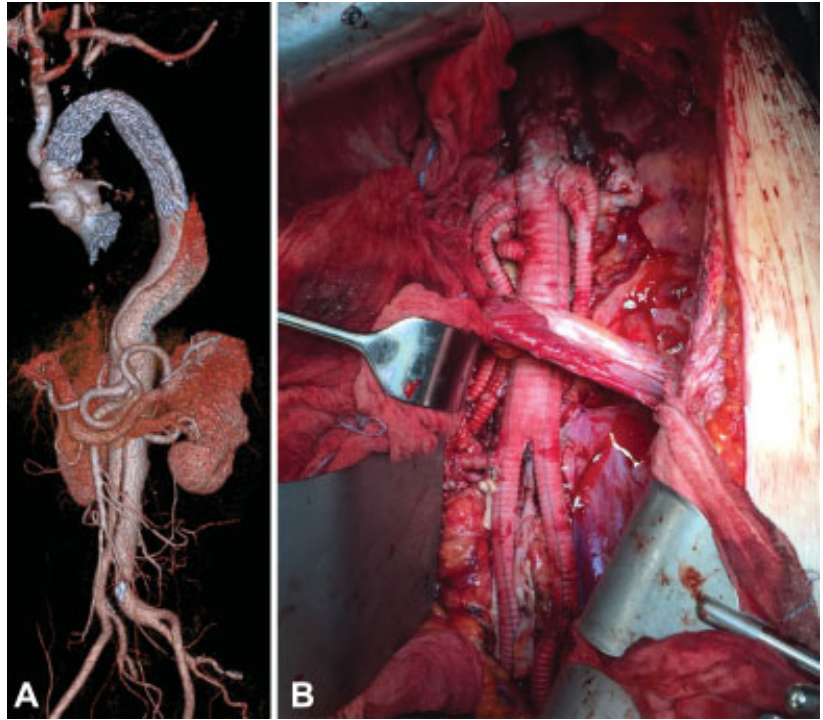

Fig. 6 Volume-rendered computed tomography reconstruction in a Marfan patient: (A) first step: aortic arch and descending aorta replacement and $(B)$ second step: thoracoabdominal aortic replacement.

\section{Discussion}

In patients with chronic dissection, aortic repair is usually performed electively for aneurysmal degeneration of the false lumen. Most of these patients are survivors of De Bakey's Type- 1 dissections. After repair of acute Type-A aortic dissection by graft replacement of the ascending aorta, the false lumen distal to the aortic graft remains patent in 70 to $100 \%$ of patients. ${ }^{20}$ Follow-up has shown that reoperation for aneurysmal dilatation of residual dissected aorta is required in 20 to $30 \%$ of patients within the first 5 to 7 years. The need for reoperation is more common in patients with Marfan's syndrome and when the original intimal tear involved the aortic arch at the time of the first operation. ${ }^{3-6}$

For this indication, it is not only important to cover the proximal entry tear; one must also address all-entry tears feeding the aneurysmal segment of the aorta. Most commonly, this aneurysmal degeneration occurs in the distal aortic arch and the proximal descending aorta, making the FET technique particularly well suited for this indication. The rationale for using a stent graft in ChAD is the proximal expansion of the true lumen covering the reentry sites along the proximal descending thoracic aorta, as well as the realization of a satisfactory landing zone, for a possible future distal aortic reoperation.

As FET has evolved, many variations for implantation have been described. ${ }^{20-23}$ Their common feature is that the stent graft is delivered into the open aorta under circulatory arrest and sutured into position. However, this necessitates suturing the stent graft, at least partially, into the dissected aortic arch. Although these are useful techniques, marked aneurysmal dilatation of the proximal descending thoracic aorta, the usual site for attachment of the aortic graft, is often present in the setting of ChAD and may preclude safe anastomosis of a graft to the aorta in this area. Furthermore, all hybrid grafts retain the complexities of surgery and its associated risks, particularly the prolonged ischemic durations of the heart, brain, spinal cord, and viscera. ${ }^{24,25}$

We have described a new surgical approach for the treatment of chronic aortic arch and proximal descending aortic dissection following repair of a Type-A acute aortic dissection. The advantage of our technique is that, after releasing the stent graft under circulatory arrest, we secure it to the graft previously implanted in the ascending aorta in without touching the native dissected aorta. The performance of an anastomosis in Hishimaru's zone 0 presents an easy surgical view and an enhanced suture control. Consequently, we have observed fewer hemorrhagic complications with greater hemodynamic stability in the early postoperative period. Other advantages of this technique include the short circulatory arrest time and, thus, a theoretically decreased likelihood of spinal cord damage. In our initial experience, we did not observe any neurologic complications. Another advantage is the avoidance of a potential injury to the recurrent laryngeal nerve.

During conventional surgery for AAD, a short ascending aortic replacement is generally performed, without cerebral vessel debranching and arch replacement. In repeat surgery, this surgical graft rarely provides enough room to perform cerebral vessel debranching on beating heart and to obtain a reliable landing zone for a stable TEVAR. Length of the ascending aorta Dacron graft less than $5 \mathrm{~cm}$ was considered a contraindication to performing a total cerebral vessel rerouting on beating heart followed by a TEVAR.

Recently, we have extended this surgical strategy not only for the treatment of ChAD but also for the treatment of arch and proximal descending thoracic aorta aneurysms for one step repair or as a bridge to definitive thoracoabdominal repair.

\section{Conclusions}

This study documents our experience with a small number of patients and a short follow-up period. However, our data suggest that this modified FET technique without "touching" the native dissected aorta constitutes a suitable alternative to a more complex surgery such as the staged ET approach or the classical FET technique with the distal anastomosis in the descending thoracic aorta. Our study may represent a less demanding solution to redo Type-A aortic dissection surgery in which substantial enlargement of the proximal descending thoracic aorta precludes an easy suturing of a prosthetic graft to this segment of the aorta. This new technique is now our preferred method of management. A larger number of patients and longer follow-up will be required to confirm these initial encouraging results.

Funding

None.

Conflict of Interest

The authors declare no conflict of interest related to this article. 


\section{Acknowledgment}

None.

\section{References}

1 Andersen ND, Williams JB, Hanna JM, Shah AA, McCann RL, Hughes GC. Results with an algorithmic approach to hybrid repair of the aortic arch. J Vasc Surg 2013;57(03):655-667, discussion 666-667

2 David TE. Surgery for acute type A aortic dissection. J Thorac Cardiovasc Surg 2015;150(02):279-283

3 Bachet JE, Termignon JL, Dreyfus G, et al. Aortic dissection. Prevalence, cause, and results of late reoperations. J Thorac Cardiovasc Surg 1994;108(02):199-205, discussion 205-206

4 Kobuch R, Hilker M, Rupprecht L, et al. Late reoperations after repaired acute type A aortic dissection. J Thorac Cardiovasc Surg 2012;144(02):300-307

5 Halstead JC, Meier M, Etz C, et al. The fate of the distal aorta after repair of acute type A aortic dissection. J Thorac Cardiovasc Surg 2007;133(01):127-135

6 Rylski B, Bavaria JE, Beyersdorf F, et al. Type A aortic dissection in Marfan syndrome: extent of initial surgery determines long-term outcome. Circulation 2014;129(13):1381-1386

7 Estrera AL, Miller CC III, Porat EE, Huynh TT, Winnerkvist A, Safi HJ. Staged repair of extensive aortic aneurysms. Ann Thorac Surg 2002;74(05):S1803-S1805, discussion S1825-S1832

8 Heinemann MK, Buehner B, Jurmann MJ, Borst HG. Use of the "elephant trunk technique" in aortic surgery. Ann Thorac Surg 1995;60(01):2-6, discussion 7

9 Di Bartolomeo R, Murana G, Di Marco L, et al. Frozen versus conventional elephant trunk technique: application in clinical practice. Eur J Cardiothorac Surg 2017;51(Suppl 1):i20-i28

10 Castrovinci S, Murana G, de Maat GE, et al. The classic elephant trunk technique for staged thoracic and thoracoabdominal aortic repair: long-term results. J Thorac Cardiovasc Surg 2015;149(02): 416-422

11 Bavaria J, Vallabhajosyula P, Moeller P, Szeto W, Desai N, Pochettino A. Hybrid approaches in the treatment of aortic arch aneurysms: postoperative and midterm outcomes. J Thorac Cardiovasc Surg 2013;145(3, Suppl):S85-S90

12 Kavanagh EP, Jordan F, Hynes N, et al. Hybrid repair versus conventional repair for aortic arch dissection. Cochrane Database Syst Rev 2018;1:CD012920. doi: 10.1002/14651858.CD012920
13 Chen IM, Shih C-C. Extending hybrid approach to residual Stanford type A dissecting aortic aneurysm. Interact Cardiovasc Thorac Surg 2008;7(05):794-796

14 Bavaria J, Milewski RK, Baker J, Moeller P, Szeto W, Pochettino A. Classic hybrid evolving approach to distal arch aneurysms: toward the zone zero solution. J Thorac Cardiovasc Surg 2010;140(6, Suppl):S77-S80, discussion S86-S91

15 Kato M, Ohnishi K, Kaneko M, et al. New graft-implanting method for thoracic aortic aneurysm or dissection with a stented graft. Circulation 1996;94(9, Suppl):II188-II193

16 Karck M, Chavan A, Hagl C, Friedrich H, Galanski M, Haverich A. The frozen elephant trunk technique: a new treatment for thoracic aortic aneurysms. J Thorac Cardiovasc Surg 2003;125(06): 1550-1553

17 Jakob H, Dohle D, Benedik J, et al. Long-term experience with the E-vita Open hybrid graft in complex thoracic aortic disease. Eur J Cardiothorac Surg 2017;51(02):329-338

18 Di Eusanio M, Pantaleo A, Murana G, et al. Frozen elephant trunk surgery-the Bologna's experience. Ann Cardiothorac Surg 2013;2 (05):597-605

19 Matalanis G, Perera NK, Galvin SD. Aortic arch replacement without circulatory arrest or deep hypothermia: the "branchfirst" technique. J Thorac Cardiovasc Surg 2015;149(2, Suppl): S76-S82

20 Malvindi PG, van Putte BP, Sonker U, Heijmen RH, Schepens MA, Morshuis WJ. Reoperation after acute type a aortic dissection repair: a series of 104 patients. Ann Thorac Surg 2013;95(03):922-927

21 Roselli EE. Trade in the hammer for a power driver-perspectives on the frozen elephant trunk repair for aortic arch disease. Ann Cardiothorac Surg 2013;2(05):633-639

22 Sun LZ, Ma WG, Zhu JM, et al. Sun's procedure for chronic type A aortic dissection: total arch replacement using a tetrafurcate graft with stented elephant trunk implantation. Ann Cardiothorac Surg 2013;2(05):665-666

23 Roselli EE, Isabella MA. Frozen Elephant trunk procedure. Optechs Thoracic Cardiovasc Surg 2013;18(02):87-100

24 Katayama K, Uchida N, Katayama A, et al. Multiple factors predict the risk of spinal cord injury after the frozen elephant trunk technique for extended thoracic aortic disease. Eur J Cardiothorac Surg 2015;47(04):616-620

25 Di Eusanio M, Pantaleo A, Murana G, et al. Frozen elephant trunk surgery-the Bologna's experience. Ann Cardiothorac Surg 2013;2 (05):597-605 